\title{
Estudio cronoamperométrico de la transferencia de triazinas a través de la interfase de dos soluciones electrolíticas inmiscibles
}

\author{
Chronoamperometric studies of triazines transfer across the interface of two \\ immiscible electrolyte solutions
}

\author{
Cervando Sánchez Muñoz ${ }^{1 *} \quad$ Rosario Enríquez Rosado ${ }^{1} \quad$ Héctor López Arjona $^{2}$ \\ Miguel Velázquez Manzanares ${ }^{3}$
}

Recibido 22 de junio de 2016, aceptado 21 de enero de 2018

Received: June 22, 2016 Accepted: January 21, 2018

\begin{abstract}
RESUMEN
En este trabajo, se estudió la transferencia de los herbicidas ametrina, atrazina y prometrina a través de la interfase de dos soluciones electrolíticas inmiscibles (agual1,2-dicloroetano) mediante las técnicas de voltamperometría cíclica y cronoamperometría en una celda electroquímica de vidrio de cuatro electrodos. Los experimentos se llevaron a cabo en condiciones ácidas para asegurar la protonación de la molécula, a través de los voltamperogramas cíclicos se determinó el potencial de transferencia para cada una de las triazinas estudiadas, mismo que sirvió para realizar los estudios cronoamperométricos. Estos últimos mostraron valores de coeficientes de difusión del orden de $10^{-6} \mathrm{~cm}^{2} \mathrm{~s}^{-1}$, los cuales están dentro de los parámetros obtenidos por voltamperometría cíclica. Los resultados sugieren que las triazinas se mueven más rápido en la fase acuosa que en la fase orgánica. Así, desde un punto de vista ambiental, estos resultados ayudan a comprender el transporte de este tipo de compuestos a través de un sistema bifásico, como lo es la membrana-agua de una célula biológica.
\end{abstract}

Palabras clave: Herbicidas, interfase agua1,2-dicloroetano, cronoamperometría, coeficientes de difusión.

\begin{abstract}
In this work, the transfer of ametryn, prometryn and atrazine herbicides was studied through the interface of two immiscible electrolyte solutions (water 1,2-dichloroethane) using cyclic voltammetry and chronoamperometry techniques in a four electrode configuration cell. The experiments were carried out under acidic conditions to ensure protonation of the molecule and the formal transfer potential for the three herbicides was measured. The chronoamperometric results showed that diffusion coefficients values are in order of $10^{-6} \mathrm{~cm}^{2} \mathrm{~s}^{-1}$, which are within the parameters obtained by cyclic voltammetry. The results suggest that the triazines move faster in the aqueous phase than in the organic phase. Thus, from an environmental point of view, these results could help to understand the transport of these compounds through a two-phase system, such as the membrane water from a biological cell.
\end{abstract}

Keywords: Herbicide, water 1,2-dichloroethane interface, chronoamperometry, diffusion coefficients.

1 Instituto de Ecología. Universidad del Mar. Ciudad Universitaria s/n, Puerto Ángel, C. P. 70902. San Pedro Pochutla, México. E-mail: cervandosm@gmail.com; r.enriquez4@gmail.com

2 Universidad del Papaloapan. Circuito Central, No. 200, Col. Parque Industrial C. P. 68301. Tuxtepec, México. E-mail: hectorlopez189@hotmail.com

3 Facultad de Ciencias Químicas. Universidad Autónoma de Coahuila. Blvd. V. Carranza S/N, 25280. Saltillo, México. E-mail:mvmiguel@hotmail.com

* Autor de correspondencia: cervandosm@gmail.com 


\section{INTRODUCCIÓN}

Los herbicidas se utilizan para eliminar la maleza y aumentar el rendimiento de la producción de alimentos. La Comisión Intersecretarial para el Control del Proceso y uso de Plaguicidas, Fertilizantes y Sustancias Tóxicas (CICOPLAFEST) es la responsable de la elaboración y actualización del catálogo de plaguicidas permitidos en México. La ametrina, atrazina y prometrina, son herbicidas que pertenecen a la familia de las triazinas; la ametrina y prometrina pertenecen al grupo de las metiltiotriazinas, mientras que la atrazina se clasifica en el grupo de las clorotriazinas. Los cultivos en dónde se permite la aplicación de éstos herbicidas según la CICOPLAFEST se muestran en la Tabla 1; históricamente, la atrazina es la triazina más utilizada [1]. Debido a su persistencia en el ambiente, las triazinas se encuentran en suelo y agua superficial; también se han encontrado en agua potable [2]. Estas moléculas son hidrofóbicas y pueden atravesar las membranas de las células biológicas ya sea en su forma protonada o neutra, por esta razón, surge el interés por estudiar la transferencia de estos herbicidas en un sistema bifásico (agualsolvente orgánico) [3].

Tabla 1. Cultivos permitidos para aplicar ametrina, atrazina y prometrina [6].

\begin{tabular}{|c|c|l|}
\hline Triazina & \multicolumn{1}{c|}{ Cultivos } \\
\hline Ametrina & $\begin{array}{l}\text { Caña de } \\
\text { azúcar, maíz, } \\
\text { naranjo, } \\
\text { papa, piña, } \\
\text { plátano y } \\
\text { toronjo }\end{array}$ \\
\hline Prometrina & $\begin{array}{l}\text { Caña de } \\
\text { azúcar, maíz, } \\
\text { piña y sorgo }\end{array}$ \\
\hline
\end{tabular}

La Interfase de Dos Soluciones Electrolíticas Inmiscibles (IDSEI) es una herramienta importante para comprender el transporte de iones y moléculas orgánicas en un sistema que presenta una frontera similar a un ambiente hidrofóbico [4]. La IDSEI, es un sistema en donde se puede estudiar, desde el punto de vista electroquímico, la transferencia de moléculas de importancia ambiental como son las triazinas a través de la interfase, utilizando para ello diferentes técnicas electroquímicas (voltamperometría cíclica y cronoamperometría, por ejemplo). La IDSEI se forma al poner en contacto dos solventes con sus respectivos electrolitos. Generalmente se trata de agua y un solvente orgánico ligeramente polar con moderada o alta permisibilidad dieléctrica como el nitrobenceno o 1,2-dicloroetano [5].

Los estudios de los procesos electroquímicos en la IDSEI, han recibido especial atención debido a su amplio rango de aplicación en sistemas químicos y biológicos [7]. Una característica de la IDSEI consiste en la diversidad de las reacciones de transferencia de carga que pueden estudiarse por diferentes técnicas electroquímicas. Los tipos de transferencia de carga a través de la IDSEI se pueden clasificar en: i) transferencia iónica simple, ii) transferencia de carga facilitada por ionóforos y iii) transferencia de electrones [8]. Cuando una carga se transfiere a través de la IDSEI, la corriente observada con las diferentes técnicas corresponde a los procesos difusionales de la especie cargada a través de la interfase [9]. En la IDSEI se han abordado estudios respecto a la transferencia de especies iónicas desde un punto de vista electroanalítico, básicamente estos se han realizado en interfases pequeñas; por ejemplo, el estudio del comportamiento de la ractopamina a través de un arreglo microinterfacial (microIDSEI) investigado por voltamperometría cíclica y voltamperometría de barrido lineal [10], así como estudios de $\boldsymbol{\beta}$-bloqueadores en interfases del orden de los nanoamperios (nanoIDSEI) [11]. En el caso de las triazinas destacan los trabajos desarrollados para la determinación de algunas triazinas, como la prometrina [12] así como estudios realizados en interfases modificadas con una monocapa de lípidos, en la que se abordó el efecto de esta en el transporte de la molécula metamitrona a través de la interfase agual1,2-dicloroetano [13].

El objetivo de este trabajo fue determinar los coeficientes de difusión para ametrina, atrazina y prometrina a través de la interfase agual 1,2-dicloroetano a través de estudios de cronopotenciometría. 


\section{METODOLOGÍA}

Las triazinas utilizadas fueron de grado analítico (Sigma Aldrich). Se utilizó 1,2-dicloroetano (Gold Label, Aldrich) como fase orgánica, mientras que en la fase acuosa se utilizó agua ultrapura obtenida mediante un equipo de agua ultrapura (Easypure UV, Barnnstead). Las sales que se emplearon como electrolito soporte fueron el cloruro de litio (Sigma Aldrich) para la fase acuosa y tetrakis 4-clorofenilborato de tetrafenilarsénico (TPAsTPBCl) para la fase orgánica, el pH se ajustó con $\mathrm{H}_{2} \mathrm{SO}_{4}(\mathrm{~J}$. T. Baker) a pH 1.0 ó 2.0 , el valor de $\mathrm{pH}$ siempre estuvo por debajo del $\mathrm{p} K a$ de la triazina estudiada.

Las técnicas de voltamperometría cíclica y cronoamperometría se realizaron en un potenciostato/ galvanostato (Autolab, PGSTAT30, Eco CHEMIE Holanda), utilizando la configuración electrónica de cuatro electrodos. Los datos se almacenaron por medio del programa GPES versión 4.9 en una computadora de escritorio con un procesador Pentium III. Para los estudios electroquímicos se empleó una celda electroquímica de vidrio con cuatro compartimentos. Se utilizaron cuatro electrodos, dos contraelectrodos de platino (Sigma-Aldrich), uno en cada fase y dos electrodos de referencia de Calomel Saturados (SCE, por sus siglas en inglés) uno en cada fase. Estos últimos a través de un capilar Luggin se proyectaron hasta la interfase formada por los dos solventes, cada uno en su fase respectiva, el área de contacto entre los dos líquidos fue de $0.2 \mathrm{~cm}^{2}$. La configuración de la celda se muestra a continuación:

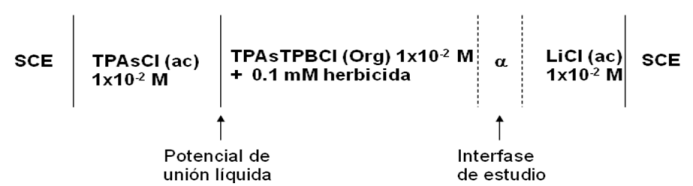

Los potenciales aplicados fueron transformados a la escala de Galvani de acuerdo a [3].

\section{RESULTADOS Y DISCUSIÓN}

En estudios previos se observó que la familia de este tipo de moléculas son susceptibles de protonarse y transferirse completamente a través de la IDSEI en condiciones de $\mathrm{pH}<\mathrm{pKa}$ [3]. Por lo que en el presente trabajo, se consideró un valor de $\mathrm{pH}$ de la fase acuosa por debajo del valor de $\mathrm{p} K$ a de las triazinas estudiadas, esto es a pH 1.0 y 2.0. Para ello el $\mathrm{pH}$ de la fase acuosa se modificó. El equilibrio ácido base para este tipo de moléculas en un sistema bifásico, es el siguiente:

$$
\mathrm{A}(\mathrm{o}) \stackrel{\mathrm{I}}{\rightleftarrows} \mathrm{A}(\mathrm{a})+\mathrm{H}^{+}(\mathrm{a}) \stackrel{\mathrm{I}}{\rightleftarrows} \mathrm{AH}^{+}(\mathrm{o}) \stackrel{\mathrm{III}}{\rightleftarrows} \mathrm{AH}^{+}(\mathrm{a})
$$

donde $\mathrm{A}$ es el herbicida en su forma neutra, $\mathrm{AH}^{+}$es el herbicida en su forma protonada, a y o son la fase acuosa y orgánica, respectivamente. De acuerdo con los diagramas de partición para este grupo de triazinas, reportados con anterioridad [3], a $\mathrm{pH}<\mathrm{p} K a$ el equilibrio termodinámico se desplaza lo suficiente para inducir la transferencia de la especie cargada a través de la IDSEI. En estas condiciones la especie predominante es la forma protonada del herbicida, su transferencia se puede describir de la siguiente forma

$$
\mathrm{AH}^{+}(\mathrm{a}) \stackrel{I I I}{\rightleftarrows} \mathrm{AH}^{+}(\mathrm{o})
$$

a $\mathrm{pH}>\mathrm{p} K a$ el herbicida se encuentra en su forma neutra y predominará en la fase orgánica [3].

En la Figura 1 se muestran los voltamperogramas cíclicos (VC) de la transferencia de la ametrina, atrazina y prometrina. En la Figura 1 inciso a) la línea punteada representa la señal del electrólito soporte (línea base), que corresponde al TPAsTPBCl a una concentración de $0.01 \mathrm{M}$ en la fase orgánica y $\mathrm{LiCl} 0.01 \mathrm{M}$ en la fase acuosa, esta última con un $\mathrm{pH}$ ajustado a 2.0, la velocidad de polarización fue de $50 \mathrm{mV} / \mathrm{s}$. La línea en donde no se observa corriente corresponde a la ventana de potencial, y se puede decir que es la región polarizable donde no ocurre una reacción faradaíca atribuida al electrolito soporte.

El analito se inyectó en la fase orgánica y se dejó que el sistema se redistribuyera por un espacio de 20 minutos, posteriormente la interfase se polarizó. En la Figura 1, incisos b, c y d se muestran los VC para la transferencia de ametrina, atrazina y prometrina. Como se puede ver, la señal de transferencia de los herbicidas se distingue de la línea base. Cuando el barrido se realiza a potenciales positivos, se puede observar un pico de corriente positiva en el VC, lo que indica que la ametrina (Figura 1, inciso b) en su forma protonada se transfiere de la fase acuosa a la fase orgánica. Cuando el barrido de potencial se invierte se observa un pico de corriente negativa lo cual indica que la ametrina se trasfiere de la fase orgánica a la fase acuosa. Para el caso de las otras dos triazinas (atrazina y prometrina) se observaron comportamientos electroquímicos similares, y están acordes a los obtenidos previamente [3,14]. 

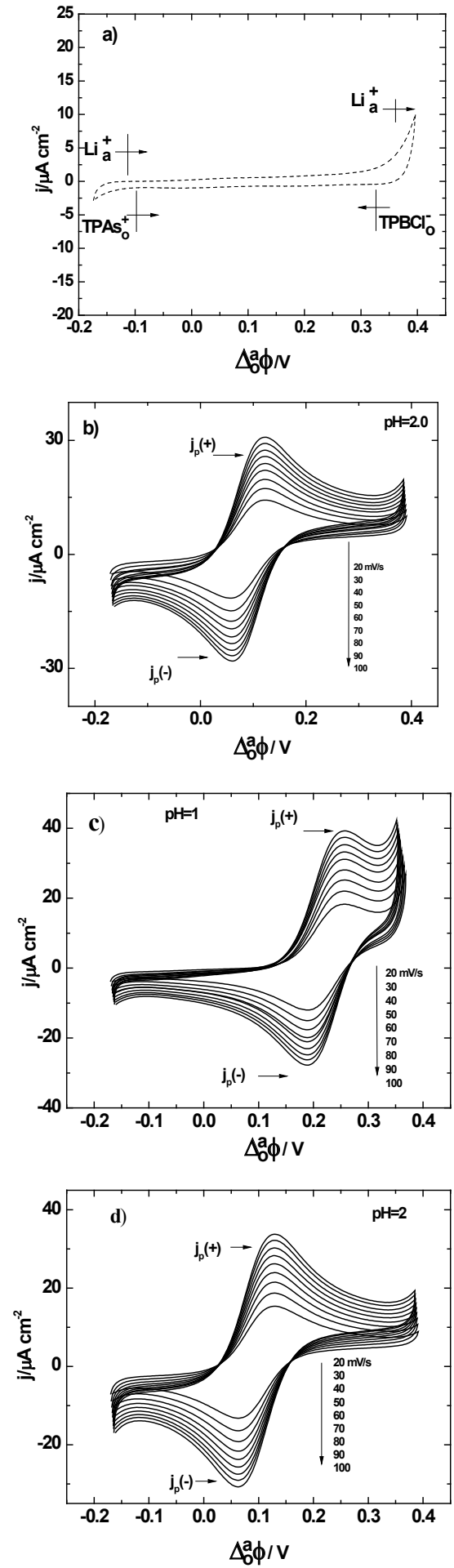

Figura 1. Voltamperogramas cíclicos. a) Línea base, b) transferencia de ametrina $(0.1 \mathrm{mM}), \mathrm{c})$ transferencia de atrazina $(0.1 \mathrm{mM})$ y d) transferencia de prometrina $(0.1 \mathrm{mM})$ a diferentes velocidades de polarización.
Los picos de corriente positivos y negativos generados a diferentes velocidades de polarización para los tres herbicidas son proporcionales a la raíz cuadrada de la velocidad de la polarización, lo que indica que la señal de corriente observada es un proceso dominado por la difusión de la especie protonada en la fase acuosa como en la fase orgánica. Mientras que la separación entre el pico de corriente positivo y negativo correspondiente a la transferencia de las especies fue de $60 \mathrm{mV}$ para la ametrina, $68 \mathrm{mV}$ para la atrazina y $64 \mathrm{mV}$ para la prometrina; lo que prueba que la transferencia de estas moléculas es un proceso reversible, ya que la diferencia de potencial de los picos de corriente es cercano al valor teórico de 59 $\mathrm{mV}$ predicha por la ecuación de Nernst para sistemas reversibles.

Los valores de potenciales de onda media $\left(\Delta_{o}^{a} \phi_{1 / 2}\right)$ se obtuvieron de los $\mathrm{VC}$ con valores de $90 \mathrm{mV}, 220$ $\mathrm{mV}$ y $93 \mathrm{mV}$ para la ametrina, atrazina y prometrina, respectivamente.

\section{Estudios cronoamperométricos}

En la Figura 2, se muestran cronoamperogramas para valores de potencial por debajo y arriba del $\Delta_{o}^{a} \phi_{1 / 2}$ correspondiente a la transferencia del herbicida a través de la interfase agual 1,2-dicloroetano. En los cronoamperogramas para la transferencia de ametrina se aprecia que la corriente límite positiva y negativa de cada potencial aplicado se presentan a un mismo tiempo (Figura 2, incisos a y b), el potencial aplicado va de $26 \mathrm{mV}$ a $116 \mathrm{mV}$ en la escala de Galvani. Para los otros dos herbicidas se puede apreciar un comportamiento similar, así las señales mostradas en los cronoamperogramas de la atrazina se hicieron en 156 a $246 \mathrm{mV}$, con incrementos de $10 \mathrm{mV}$, (Figura 2 , incisos c y d) y prometrina (56 a $146 \mathrm{mV}$, con incrementos de $10 \mathrm{mV}$, Figura 2, incisos e y f).

Las curvas de corriente $v s$ tiempo reflejan el cambio del gradiente de concentración de la especie electroactiva en la vecindad de la superficie de la interfase [15], parecido al fenómeno observado en la interfase metallelectrolito. El valor de la corriente disminuye conforme transcurre el tiempo, dicho comportamiento se debe a que la concentración del herbicida cambia del lado de la interfase de la fase acuosa, ya que esta especie se transfiere a la fase contigua. Para el caso de la corriente límite positiva, al incrementar el potencial, la corriente aumenta debido a que se favorece la aproximación del herbicida hacia 
la interfase; la capa difusa acuosa sufre una expansión al incrementar la concentración en la interfase. Para el caso en donde se polariza la interfase en dirección catódica, se generan cronoamperogramas con corriente límite negativa, lo cual indica que la triazina protonada se transfiere de la fase orgánica hacia la fase acuosa. Comparando los resultados de los tres herbicidas, la atrazina es la que
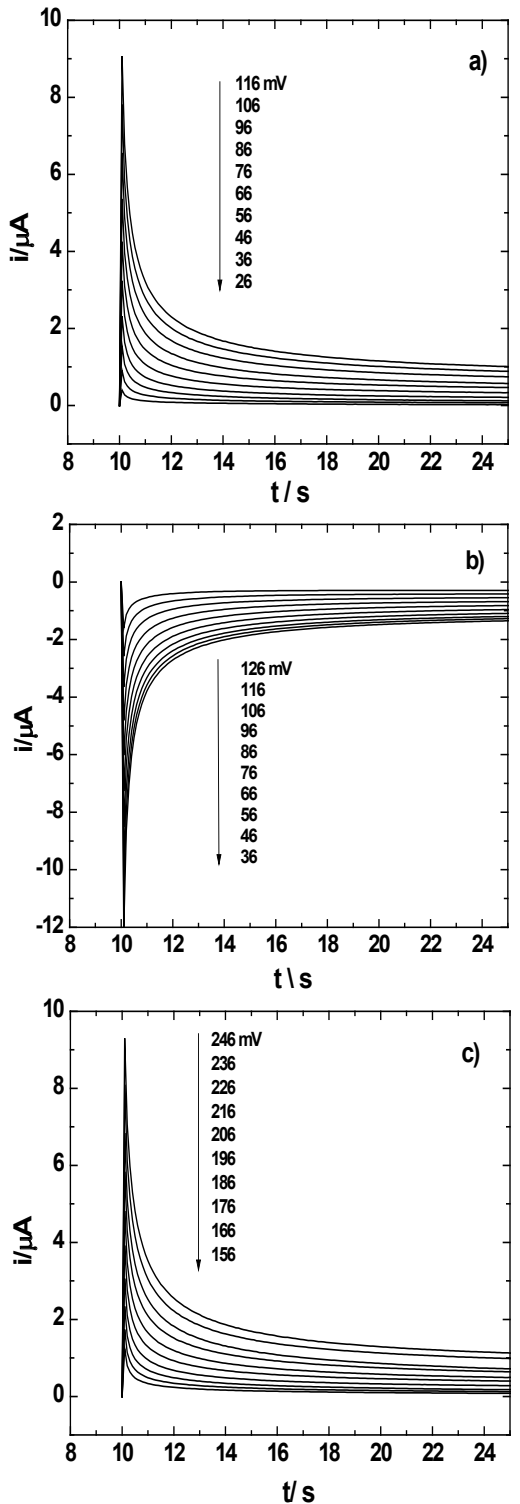

requiere un mayor potencial $(220 \mathrm{mV})$ para transferirse a través de la IDSEI en comparación con la ametrina (90 $\mathrm{mV}$ ) y prometrina $(93 \mathrm{mV})$. Este comportamiento es similar a lo observado en estudios de voltamperometría cíclica [3], es decir que en términos energéticos se requiere mayor energía para meter a la atrazina en la fase orgánica que a la ametrina o la prometrina.
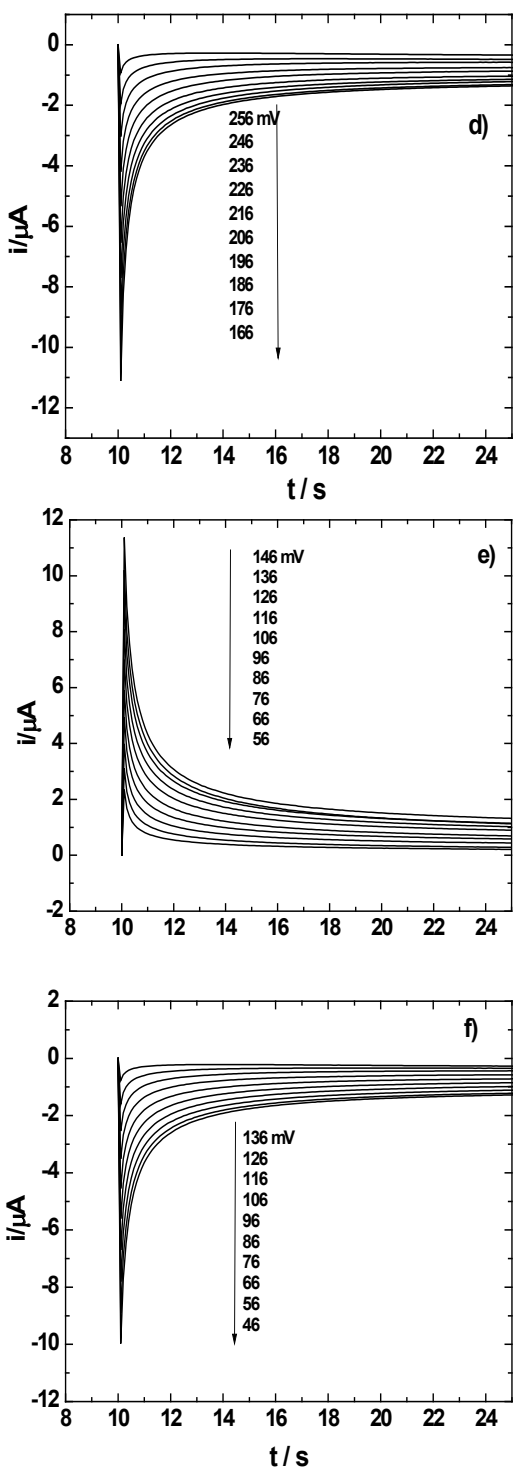

Figura 2. Cronoamperogramas para la transferencia de ametrina $(0.1 \mathrm{mM}, \mathrm{pH}=2.0$, inciso a y $\mathrm{b})$, atrazina (0.1 mM, $\mathrm{pH}=1.0$, inciso c y d) y prometrina $(0.1 \mathrm{mM}, \mathrm{pH}=2.0$, inciso e y f) a través de la interfase agual1,2-dicloroetano. Los incisos: a, c y d corresponden a la corriente límite positiva y el resto (b, d y f) a la corriente límite negativa.

Cuantitativamente la variación de la corriente $v s$ tiempo puede ser analizada mediante la ecuación de Cottrell descrita en la ecuación (3) [16]. Esta ecuación se emplea para determinar los coeficientes de difusión de especies electroactivas a partir de los resultados experimentales cronoamperométricos para estudios 
en un sistema metallsolución. Este mismo principio se puede aplicar para la interfase que se forma entre dos soluciones electrolíticas inmiscibles.

$$
i(t)=\frac{n F A D_{o}^{1 / 2} C_{o}^{*}}{\pi^{1 / 2} t^{1 / 2}},
$$

donde $i(t)$ es la corriente, $F$ es la constante de Faraday, $D$ es el coeficiente de difusión, $C$ es la
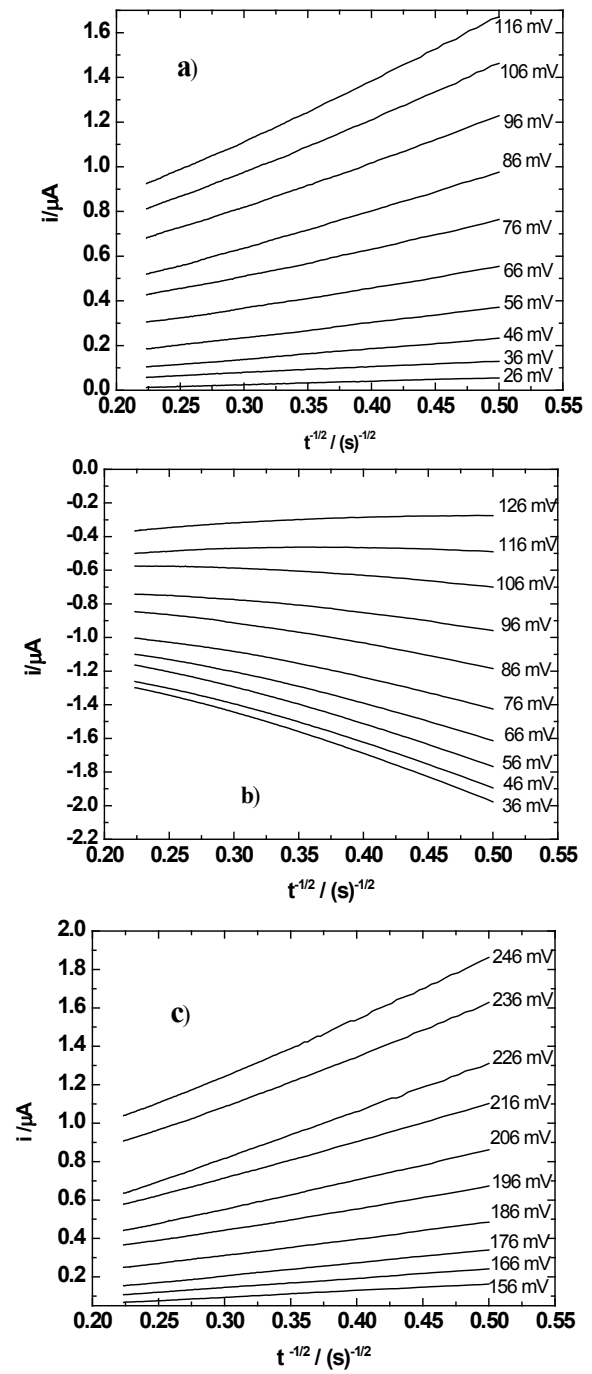

concentración del analito, $t$ el tiempo y $n$ en un sistema IDSEI representa el número de cargas transferidas.

A partir de los cronoamperogramas de la Figura 2, se graficó la corriente límite $(\mu \mathrm{A})$ en función del inverso de la raíz cuadrada del tiempo $\left(\mathrm{t}^{-1 / 2}\right)$ para generar las curvas de Cottrell que se muestran en la Figura 3; en ellas se puede ver una relación lineal entre la corriente i vs $\mathrm{t}^{-1 / 2}$ que se ajusta a la ecuación de Cottrell.
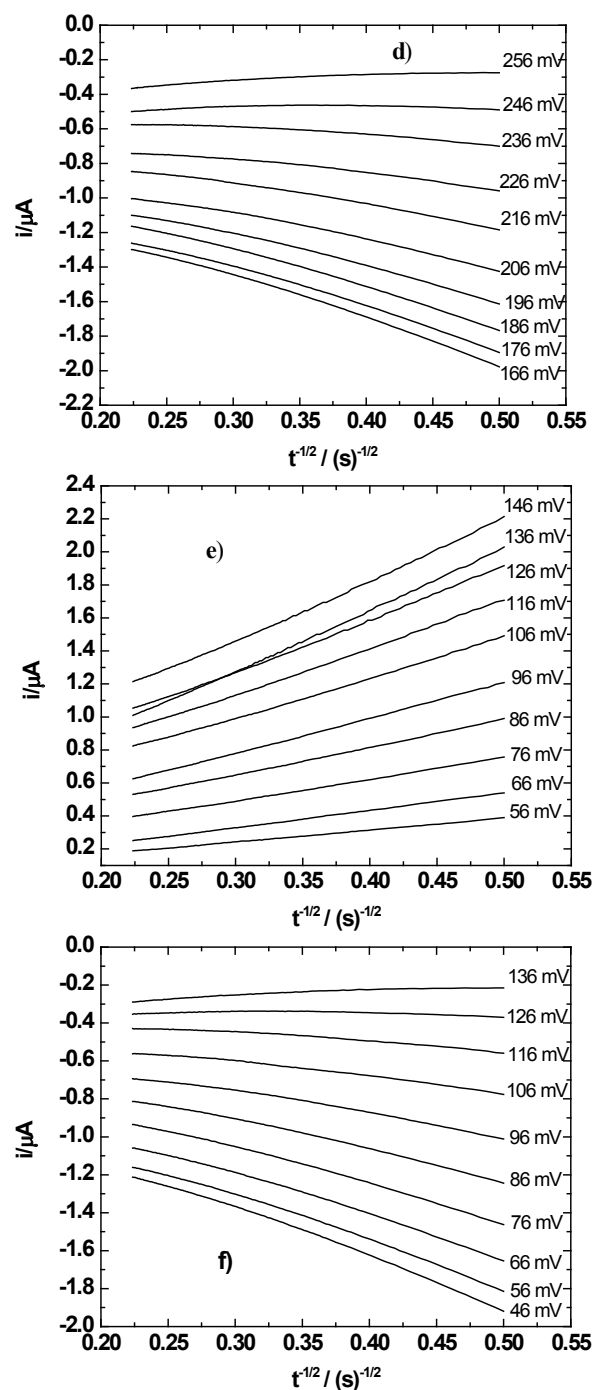

Figura 3. Curvas de Cottrell a diferentes potenciales para la transferencia de ametrina $(0.1 \mathrm{mM}, \mathrm{pH}=2.0$, inciso a y b), atrazina $(0.1 \mathrm{mM}, \mathrm{pH}=1.0$, inciso c y d) y prometrina $(0.1 \mathrm{mM}, \mathrm{pH}=2.0$, inciso e y f) a través de la interfase agual 1,2-DCE. Los incisos: a, c y d corresponden a la corriente límite positiva y el resto (b, d y f) a la corriente límite negativa.

Los coeficientes de difusión para las triazinas de interés se determinaron a potenciales cercanos al valor de potencial de onda media para cada herbicida estudiado. En términos de magnitud, los valores del coeficiente de difusión de los tres herbicidas son mayores en la fase acuosa respecto a la fase 
orgánica, esto indica que la ametrina, atrazina y prometrina se mueven rápidamente en la fase acuosa en comparación con la fase orgánica. Considerando la razón de coeficientes de difusión $\mathrm{D}^{\circ} / \mathrm{D}^{\mathrm{a}}$ evaluado en términos de la regla de Walden, esto es $\mathrm{D}^{\circ} / \mathrm{D}^{\mathrm{a}}=$ $\mathrm{h}^{\mathrm{a}} / \mathrm{h}^{\mathrm{o}}$ donde $\mathrm{h}^{\mathrm{o}} \mathrm{y} \mathrm{h}^{\mathrm{a}}$ son la viscosidad del solvente orgánico y el agua. Por lo que tenemos que $\mathrm{D}^{\%}$ $\mathrm{D}^{\mathrm{a}}=0.95$. Los valores de las constantes de difusión de la fase orgánica se aproximan al valor obtenido a través de la regla de Walden (Tabla 2, $D w r_{A H^{+}}^{o}$ ). La diferencia en los valores de los coeficientes de difusión de los herbicidas se debe a la estructura de cada una las triazinas. Para el caso de la ametrina y prometrina que pertenecen al grupo de las metiltiotriazinas, la única diferencia entre los dos herbicidas se debe a que la prometrina presenta un grupo metilo más en su estructura química. Los valores del coeficiente de difusión de los herbicidas están en el mismo orden de magnitud que los reportados en trabajos previos $[3,14]$.

El potencial de onda media se puede utilizar para determinar la energía libre de Gibbs de transferencia de la especie electroactiva de la fase acuosa hacia la fase orgánica, $\Delta_{o}^{a} G_{t, i}^{a \rightarrow o}$, de acuerdo con la siguiente ecuación

$$
\Delta_{o}^{a} \phi_{i}^{o^{\prime}}=\frac{\Delta_{o}^{a} G_{t, i}^{a \rightarrow o}}{z_{i} F}=\frac{\mu_{i}^{0, o}-\mu_{i}^{0, a}}{z_{i} F},
$$

donde $\mu_{i}^{0, o}$ y $\mu_{i}^{0, a}$ son los potenciales químicos estándar para la especie $i$ en la fase orgánica y acuosa, $\Delta_{o}^{a} \phi_{i}^{o^{\prime}}$ es el potencial formal de Galvani de transferencia para un ión $i, z_{i}$ es la carga del ión y $F$ es la constante de Faraday

$$
\Delta_{o}^{a} \phi_{1 / 2}=\Delta_{o}^{a} \phi_{i}^{o^{\prime}}+\frac{R T}{z_{i} F} \ln \left(\frac{D_{A H^{+}}^{o}}{D_{A H^{+}}^{a}}\right),
$$

donde $D_{A H^{+}}^{a}$ y $D_{A H^{+}}^{o}$ son las coeficientes de difusión del herbicida en la fase acuosa y orgánica, respectivamente (Tabla 2). A partir de la ecuaciones (4) y (5) se obtuvieron los valores de energía de Gibbs siguientes: $6.65 \mathrm{~kJ} \mathrm{~mol}^{-1}, 18.21 \mathrm{~kJ} \mathrm{~mol}^{-1} \mathrm{y}$ $6.75 \mathrm{~kJ} \mathrm{~mol}^{-1}$ para la ametrina, atrazina y prometrina, respectivamente. Estos valores indican que la transferencia de atrazina requiere mayor energía para transferirse de la fase acuosa hacia la fase orgánica en comparación con la ametrina y prometrina, estos resultados se encuentran acordes con el potencial de onda media observado en estos experimentos.
Tabla 2. Coeficientes de difusión.

\begin{tabular}{llll}
\hline Herbicida & $\begin{array}{l}\boldsymbol{D}_{A H^{+}}^{a} \\
\left(\mathbf{c m}^{2} \mathbf{s}^{-1}\right)\end{array}$ & $\begin{array}{l}\boldsymbol{D}_{\boldsymbol{A} H^{+}}^{o} \\
\left(\mathbf{c m}^{2} \mathbf{s}^{-1}\right)\end{array}$ & $\begin{array}{l}\boldsymbol{D} \boldsymbol{w} \boldsymbol{r}_{\boldsymbol{A H}}^{o} \\
\left(\mathbf{c m}^{2} \mathbf{s}^{-1}\right)\end{array}$ \\
\hline Ametrina & $3.68 \times 10^{-6}$ & $2.89 \times 10^{-6}$ & $3.49 \times 10^{-6}$ \\
Atrazina & $6.16 \times 10^{-6}$ & $5.10 \times 10^{-6}$ & $5.85 \times 10^{-6}$ \\
Prometrina & $5.55 \times 10^{-6}$ & $4.97 \times 10^{-6}$ & $5.27 \times 10^{-6}$ \\
\hline
\end{tabular}

\section{CONCLUSIONES}

Se pudo demostrar que es posible estudiar el transporte de carga a través de la interfase agual1,2dicloroetano mediante técnicas de pulsos como la cronoamperometría. Las constantes de difusión calculadas demuestran que los herbicidas tienen una velocidad mayor en la fase acuosa que en la fase orgánica. Probablemente se debe a que estas especies son más estables en la fase orgánica. En términos energéticos se observó que la atrazina requiere mayor energía para ser transferida dentro de la fase orgánica, lo cual se relaciona con su tamaño molecular.

\section{AGRADECIMIENTOS}

Se agradece al CONACYT por el apoyo financiero otorgado a través del proyecto $104427-$ SNI y a la UNPA a través del proyecto interno. C. S. M. agradece al CONACYT por la beca otorgada para la realización de estudios de posgrado.

\section{REFERENCIAS}

[1] A-J Trimble, M-J Lydy. "Effects of triazine herbicides on organophosphate insecticide toxicity in Hyalellaazteca". Archives of Environmental Contamination and Toxicology. Vol. 51 pp. 29-34. February, 2006. ISSN: 1432-0703. DOI: 10.1007/ s00244-005-0176-7.

[2] M. Farré, E. Martínez, J. Ramón, A. Navarro, J. Radjenovic, E. Mauriz, L. Lechuga, M. P. Marco, D. Barceló. "Part per trillion determination of atrazine in natural water samples by a surface plasmon resonance immunosensor". Journal of Analytical and Bioanalytical Chemistry. Vol. 388, pp. 207214. March, 2007. ISSN: 1618-2650. DOI: 10.1007/s00216-007-1214-2. 
[3] M. Velázquez-Manzanares, J. AmadorHernández, C. Cisnero-cisneros, K.A. Heredia-Lezama. "Triazine herbicides transfer at the water/1,2-dichloroethane interface". Journal of Electrochemical Society. Vol. 155, Issue 10, pp. F218-F222. August, 2008. DOI: 10.1149/1.2965795.

[4] M. Velázquez-Manzanrez. "Fundamentals and applications in electrochemistry of liquid-liquid interfaces". Pocedia Chemistry. Vol. 12, pp. 100-107. 2014. ISSN: 18766196. DOI: 10.1016/j.proche.2014.12.047.

[5] Z. Samec. "Electrochemistry at the interface between two immiscible electrolyte solutions". Pure and Applied Chemistry. Vol. 76, Issue 12, pp. 2147-2180. 2004. ISSN: 1365-3075. DOI: $10.1351 /$ pac200476122147,

[6] Comisión Intersecretarial para el Control y uso de Plaguicidas Fertilizantes y Sustancias Tóxicas (CICOPLAFEST). "Catálogo de plaguicidas". México. 2004.

[7] M. Senda, T. Kakiuchi, T. Osakai. "Electrochemistry at the interface between two inmiscible electrolyte solutions". Journal of Electrochimica Acta. Vol. 36 (2), pp. 253-262. 1991. ISSN: 0013-4686. DOI: 10.1016/0013-4686(91)85246-4.

[8] F. Reymond, D. Fermín, J-L Lee, H-H Girault. "Electrochemistry at liquid/liquid interfaces: methodology and potential applications". Journal of Electrochimica Acta. Vol. 45 (15-16), pp. 2647-2662. May, 2000. ISSN: 0013-4686. DOI: 10.1016/ S0013-4686(00)00343-1.

[9] M. Velázquez-Manzanares. "Transferencia de carga a través de la interfase de dos soluciones electrolíticas inmiscibles: fundamento y caso de estudio". Journal of the Mexican Chemical Society. Vol 47 (1), pp. 66-72. Enero-marzo, 2003. ISSN: 0583-7693.
[10] Sairi M., Arraigan D. W. M. "Electrochemical detection of ractopamine at arrays of microliquidlliquid interfaces". Talanta. Vol. 132, pp. 204-214. January, 2015. ISSN: 0039-9140. DOI: 10.1016/j.talanta.2014.08.060.

[11] Y. Liu, J. Strutwolf, D. Arrigan. "Ion-transfer voltammetric behavior of propranolol at nanoscale liquid-liquid interface arrays". AnalyticalChemistry. Vol. 87 (8) pp. 4487-4494. (2015). DOI: 10.1021/acs. analchem.5b00461

[12] A. V. Juarez, L. M. Yudi. "Quantitative analysis of prometrine herbicide by LiquidLiquid extraction procedures coupled to electrochemical measurements". Electroanalysis. Vol. 21 (6) pp. 767-771. March, 2009. DOI: 10.1002/elan.200804476.

[13] A. G. Reyes Reyes, J. Amador Hernández, M. Velázquez Manzanares. "Electrochemical Behavior of Metamitron Herbicide at the Interface of Two Immiscible Electrolyte Solutions". Journal of the Mexican Chemical Society. Vol. 58 (3), pp. 326-33. JulioSeptiembre 2014. ISSN: 1870-249X.

[14] A. V. Juarez, L. M. Yudi. "Electrochemical study of s-triazine herbicides transfer across the water/1,2-dicloroethane interface". Electroanalysis. Vol. 15, Issue 18, pp. 1481-1487. October, 2003. DOI: 10.1002/ elan.200302718

[15] J. Wang J. "Analytical Electrochemistry". Second Edition. Wiley-Vch. Second Edition. United States of America. 2000. ISBN: 0-471-22823-0.

[16] C. G. Zoski. "Handbook of electrochemistry". First edition. Elsevier, Netherlands. 2007. ISBN: 0-444-51958-0 